\title{
The perceived global impact of the COVID-19 pandemic on doctors' medical and surgical training: an international survey
}

. TMS Collaborative ${ }^{1}$, Ryan Laloo ${ }^{2}$, Rama Santhosh Karri ${ }^{3}$, Kasun Wanigasooriya ${ }^{4}$, William Beedham ${ }^{4}$, Adnan Darr ${ }^{3}$, Georgia Layton ${ }^{5}$, Peter Logan ${ }^{6}$, Yanyu Tan ${ }^{7}$, Devender Mittapalli ${ }^{8}$, Tapan Patel ${ }^{9}$, Vivaswan Dutt Mishra ${ }^{10}$, Osama Faleh Odeh ${ }^{11}$, Swathi Prakash $^{12}$, Salma Elnoamany ${ }^{13}$, Sri Ramya Peddinti ${ }^{14}$, Elorm Adzoa Daketsey ${ }^{15}$, Shardool Gadgil $^{16}$, Ahmad Bouhuwaish ${ }^{17}$, Ahmad Ozair ${ }^{18}$, Sanchit Bansal ${ }^{19}$, Muhammed Elhadi ${ }^{20}$, Aditya Amit Godbole ${ }^{21}$, Ariana Axiaq ${ }^{22}$, Faateh Ahmad Rauf ${ }^{23}$, and Ashna Ashpak ${ }^{24}$

${ }^{1}$ The Master Surgeon Trust

${ }^{2}$ Leeds General Infirmary

${ }^{3}$ Royal Wolverhampton Hospitals NHS Trust

${ }^{4}$ University of Birmingham College of Medical and Dental Sciences

${ }^{5}$ University Hospitals of Leicester NHS Trust

${ }^{6}$ Walsall Healthcare NHS Trust

${ }^{7}$ North East Deanery, United Kingdom

${ }^{8}$ University Hospitals Plymouth NHS Trust

${ }^{9}$ Baroda Medical College

${ }^{10}$ Moti Lal Nehru Medical College

${ }^{11}$ The University of Jordan Faculty of Medicine

${ }^{12}$ HCG-MSR Cancer Centre

${ }^{13}$ Menoufia University Faculty of Medicine

${ }^{14}$ Indira Gandhi Medical College and Research Institute

${ }^{15}$ Betsi Cadwaladr University Health Board

${ }^{16}$ Lokmanya Tilak Municipal Medical College and General Hospital

${ }^{17}$ Faculty of Medicine Tobruk University

${ }^{18}$ King George's Medical University

${ }^{19}$ Vardhman Mahavir Medical College and Safdarjung Hospital

${ }^{20}$ University of Tripoli Faculty of Medicine

${ }^{21}$ Bharati Vidyapeeth Deemed University Medical College

${ }^{22}$ Queen's University Belfast Faculty of Medicine Health and Life Sciences

${ }^{23}$ Combined Military Hospital Lahore Medical College

${ }^{24}$ University of Central Lancashire School of Medicine and Dentistry

March 31, 2021

\begin{abstract}
Abstract Introduction The COVID-19 pandemic has resulted in a significant burden on healthcare systems causing disruption to medical and surgical training of doctors globally. Aims and objectives This is the first international survey assessing the
\end{abstract}


perceived impact of the COVID-19 pandemic on training of doctors of all grades and specialties. Methods An online global survey was disseminated using Survey Monkeyß between 4th August 2020 and 17th November 2020. A global network of collaborators facilitated participant recruitment. Data was collated anonymously with informed consent and analysed using univariate and adjusted multivariable analysis. Results 743 doctors of median age 27 (IQR: 25-30) were included with the majority (56.8\%, $\mathrm{n}=422)$ being male. Two-thirds of doctors were in a training post $(66.5 \%, \mathrm{n}=494), 52.9 \%(\mathrm{n}=393)$ in a surgical specialty and $53.0 \%(\mathrm{n}=394)$ in low- and middle-income countries. $69.2 \%(\mathrm{n}=514)$ reported an overall perceived negative impact of the COVID-19 pandemic on their training. A significant decline was noted among non-virtual teaching methods such as face-to-face lectures, tutorials, ward-based teaching, theatre sessions, conferences, simulation sessions and morbidity and mortality meetings (p[?]0.05). Doctors from low or middle-income countries were associated with perceived inadequate supervision while performing invasive procedures under general, local or regional anaesthetic. (p[?]0.05) Conclusion In addition to the detrimental impact of the COVID-19 pandemic on healthcare infrastructure, there has been an indirect consequence of disrupted training within medical and surgical subspecialties. A focus on reconfiguration of training programs through a variety of additional resources will become imperative to reduce the long-term sequalae of COVID-19 on doctors' training.

\section{Hosted file}

Main text.pdf available at https://authorea.com/users/405034/articles/516096-the-perceivedglobal-impact-of-the-covid-19-pandemic-on-doctors-medical-and-surgical-training-aninternational-survey 